\title{
A Structurally and Functionally Common Unit in Testes and Ovaries of Medaka (Oryzias latipes), a Teleost Fish
}

\author{
Toshiya Nishimura ${ }^{a, c}$ Shuhei Nakamura ${ }^{a, d}$ Minoru Tanaka ${ }^{a-c}$ \\ a Laboratory of Molecular Genetics for Reproduction, National Institute for Basic Biology, Okazaki, ${ }^{b}$ SOKENDAI, \\ The Graduate University for Advanced Studies, Shonan Village, Hayama, and ' Graduate School of Science, \\ Nagoya University, Nagoya, Japan; ' Max Planck Institute for Biology of Aging, Cologne, Germany
}

\section{Key Words}

foxl3 $\cdot$ Germline stem cell $\cdot$ Medaka $\cdot$ Ovary $\cdot$ sox $9 b \cdot$ Testis

\begin{abstract}
Testes and ovaries are structurally and functionally different organs, but they originate from the same gonadal primordium. Here, we propose how a tissue composed of germ cells and sox 96 -expressing cells underlain by a basement membrane develops a functionally common unit that reserves sexually indifferent or unfixed germline stem cells in both the ovaries and testes of adult medaka. During testicular development, the unit expands and gives rise to lobules where germline stem cells continuously produce a tremendous amount of sperm, while the unit in the female becomes germinal cradles that represent niche areas for neo-oogenesis. A recent finding in female fox $/ 3$ mutants demonstrates that the unit can also serve as the niche for germline stem cells continuously producing sperm in the ovary. We hypothesize that this unit in medaka might correspond to the medullary cords of the gonadal primordium in amniotes and that fragmentation of the medullary cords during ovarian development may be related to the absence of germline stem cells.
\end{abstract}

(c) 2016 S. Karger AG, Basel
Ovaries and testes are morphologically and functionally different organs, producing eggs and sperm, respectively, but are derived from the same sexually indifferent gonadal primordium. Should we see sex differentiation of the gonadal primordium as completely different forms of organogenesis or is there any unit that is common between testes and ovaries? Before addressing this issue, we would like to first explain the structure of mature testes and ovaries in medaka (Oryzias latipes).

Testes in teleosts consist of tubules or lobules that are surrounded by a basement membrane (fig. 1) [Grier, 1981]. In medaka, spermatogenesis proceeds in many lobules that radiate from an efferent duct at the most proximal position (in the central part) of the testes. The distal portion of the lobule harbors germline stem cells/ spermatogonia responsible for the continuous production of sperm [Parenti and Grier, 2004; Nakamura et al., 2011]. The germ cells in the lobule are directly surrounded by Sertoli cells that express sox $9 b$, one of the orthologs of mammalian Sox9 [Klüver et al., 2005; Nakamoto et al., 2005]. Although the detailed process of lobule development has not been well analyzed, it has been suggested that sox $9 b$-expressing cells in the gonadal primordium become Sertoli cells in the lobule [Nakamura et al., 2008].

\section{KARGER}

E-Mail karger@karger.com

www.karger.com/sxd (c) 2016 S. Karger AG, Basel

$1661-5425 / 16 / 0103-0159 \$ 39.50 / 0$ 


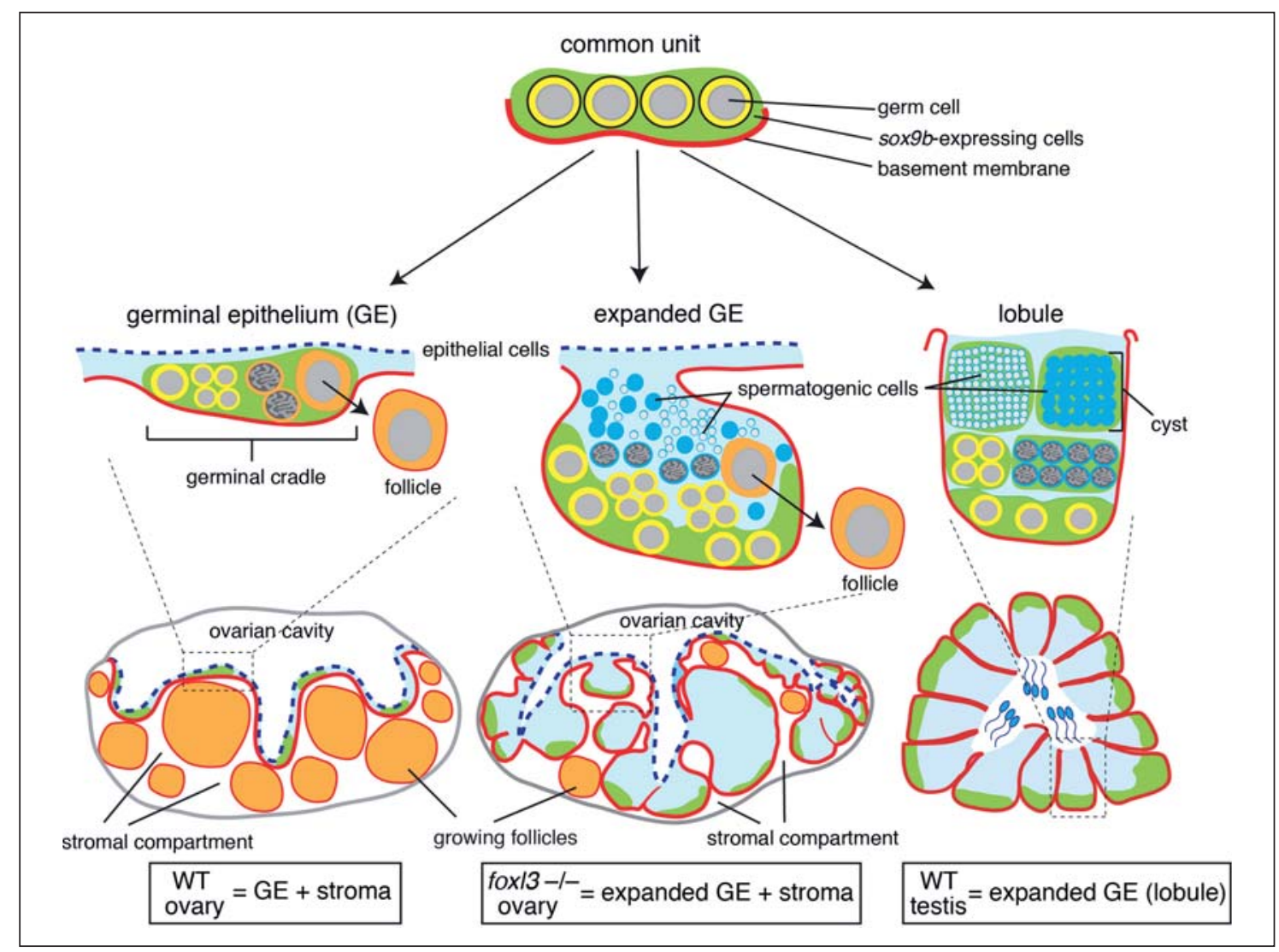

Fig. 1. The unit of testes and ovaries maintains germline stem cells in medaka. Germ cells with sox $9 b$-expressing cells (green) underlain by a basement membrane (red line) are structural and functional units common in ovaries and testes. During ovarian development, the unit, together with epithelial cells, differentiates into the germinal epithelium (GE). Within the GE, germ cells at the early stage are surrounded by sox $9 b$-expressing cells, forming germinal cradles representing a niche for germline stem cells. Diplotene oocytes formed within the germinal cradles subsequently exit into the stromal compartment where follicle formation and growth occur. In this regard, wild-type ovaries can be viewed as a

Medaka ovaries are composed of 2 main regions: a stromal compartment, where follicles grow and mature, and an ovarian cavity, into which matured oocytes are ovulated. In addition, a cellular layer, called germinal epithelium, separates the stromal compartment from the ovarian cavity. The germinal epithelium consists of epithelial cells and a basement membrane [Grier, 2002]. Within the germinal epithelium, histologically distinct units composed of sox $9 b$-expressing cells enclose germline stem cells (called germinal cradles) and serve as the place for continuous neo-oogenesis. The early stages of oogenesis, from germline stem cell proliferation to devel-
GE with a well-developed stromal compartment. In the foxl3 mutant (foxl3-/-) ovary, continuous spermatogenesis occurs from germline stem cells surrounded by sox $9 b$-expressing cells within the expanded GE. In wild-type testes, the unit of germ cells and sox9b-expressing cells expands and develops lobules in the testes. Spermatogenic cells at the same stage of development are surrounded by sox 9 b-expressing cells to form cysts, which are arranged in a distal to proximal direction. Therefore, the testis can be viewed as an expanded version of GE where spermatogenesis occurs in an arranged manner. Note that the arrangement of spermatogenesis is disorganized in the foxl3-/- ovary.

opment of diplotene oocytes, occur in the germinal epithelium, and the resultant diplotene oocytes exit into the stromal compartment to form follicles [Nakamura et al., 2010] (fig. 1).

foxl3 has recently been identified as a sperm-egg switching gene in medaka [Nishimura et al., 2015]. Disruption of foxl 3 in female medaka leads to spermatogenesis in an ovary. Along with a detailed analysis of mutant ovaries with disrupted $f \circ x l 3$, we describe how a specific histological unit, germ cells with sox $9 b$-expressing cells underlain by a basement membrane, leads to the development of sexually dimorphic structures in both the ovary 
and testis. This unit also provides us with insights into how different structures of testes and ovaries can be uniformly understood in both amniotes and medaka.

\section{Comparison of Wild-Type and fox/3 Mutant Ovaries}

The function of foxl3 is to repress spermatogenesis in the germ cells of female medaka. In female foxl3 mutants, ovaries develop normally with an ovarian cavity and a stromal compartment but spermatogenesis occurs within the germinal epithelium. Expression of nanos2, a marker of germline stem cells [Aoki et al., 2009; Beer and Draper, 2013], is detected in a subset of germ cells enclosed by sox $9 b$-expressing cells in the germinal epithelium of the foxl3 mutant ovaries [Nishimura et al., 2015]. These observations suggest that the mutant ovaries possess germinal cradles harboring germline stem cells and sustain continuous spermatogenesis within the germinal epithelium. Unfortunately, the ovarian structure does not develop a ductal system that conveys sperm to the outside of the ovary, which results in the expansion of germinal epithelium filled with sperm (fig. 1).

Occasionally in aged mutant medaka, oocytes appear between spermatogenic cells within the expanded germinal epithelium. These oocytes seem to exit into the stromal compartment as a few follicles can be observed in the stromal compartment (fig. 1). Interestingly, the follicles can mature and be ovulated to produce fertilizable eggs [Nishimura et al., 2015]. This finding supports our notion that female foxl 3 mutants develop a functional ovary comparable to an ovary of wild-type female medaka.

All these observations suggests that the unit consisting of germ cells and sox $9 b$-expressing cells, equivalent to germinal cradles in the wild-type ovary, has the potential function to maintain germline stem cells that can develop into both eggs and sperm. In the next section, we compare the gonadal structure in foxl 3 mutant ovaries with that in wild-type testes.

\section{Comparison of Wild-Type Testes and fox/3 Mutant Ovaries}

Within each lobule, sox $9 b$-expressing cells surrounding the same stage of spermatogenic cells form a developmental unit referred to as a cyst [Grier, 1981; Nakamura et al., 2011; Nishimura and Tanaka, 2014]. The different stages of these units in the lobule are arranged in a distal

A Common Unit in Testes and Ovaries of Medaka (peripheral)-proximal (central) direction such that cysts with germline stem cells/spermatogonia are located at the most distal tip of the lobules, and the cysts with spermatocytes are positioned proximal to the germline stem cell cysts. In sites closer to an efferent duct, the cysts undergoing spermatogenesis and maturation of sperm are present, and the resultant sperm are released into the efferent duct located at the most proximal position of the lobule (fig. 1) [Grier, 1976, 1981].

Within the expanded germinal epithelium in foxl3 mutant ovaries where spermatogenesis proceeds, all the developmental stages of spermatogenic cells are present. Some cysts are surrounded by sox $9 b$-expressing cells, but they are not arranged in a developmentally ordered way as seen in wild-type testes. On the inner side of the expanded germinal epithelium, spermatids and spermatocytes are found free from the close contact with sox $9 b$ expressing cells. Only early spermatogenic cells, including germline stem cells, have direct contact with sox $9 b$-expressing cells (fig. 1).

The foxl3 mutant ovaries do not express the typical male somatic marker genes, $d m r t 1$ and $p 45011 \beta$, but express the female marker genes, foxl 2 and aromatase [Kurokawa et al., 2007; Nishimura et al., 2015]. Therefore, it is likely that sox $9 b$-expressing cells in the foxl3 mutant ovaries are female cells. Unlike in amniotes, medaka sox $9 b$ is not directly involved in male development of the gonad [Nakamura et al., 2012]. The lack of male-specific expression in the sox $9 b$-expressing cells may explain the reason for both the developmentally disorganized positioning of spermatogenic cells and reduced contact of germ cells with sox $9 b$-expressing cells.

In medaka, isolated primary spermatocytes can differentiate into matured sperm without interaction with somatic cells in vitro [Saiki et al., 1997]. Therefore, the primary spermatocytes produced in the foxl 3 mutant ovaries may cell-autonomously differentiate into sperm. The close contact of germ cells with sox $9 b$-expressing cells in the cysts of wild-type testes may be important for the arrangement of spermatogenesis but not for spermatogenesis itself.

\section{A Common Unit Found in Ovaries and Testes}

These observations suggest that a structural unit, comprising sox $9 b$-expressing cells and germ cells underlain by a basement membrane, is present in wild-type ovaries, testes, and foxl 3 mutant ovaries (fig. 1) and shares the function of the maintenance of germline stem cells in 


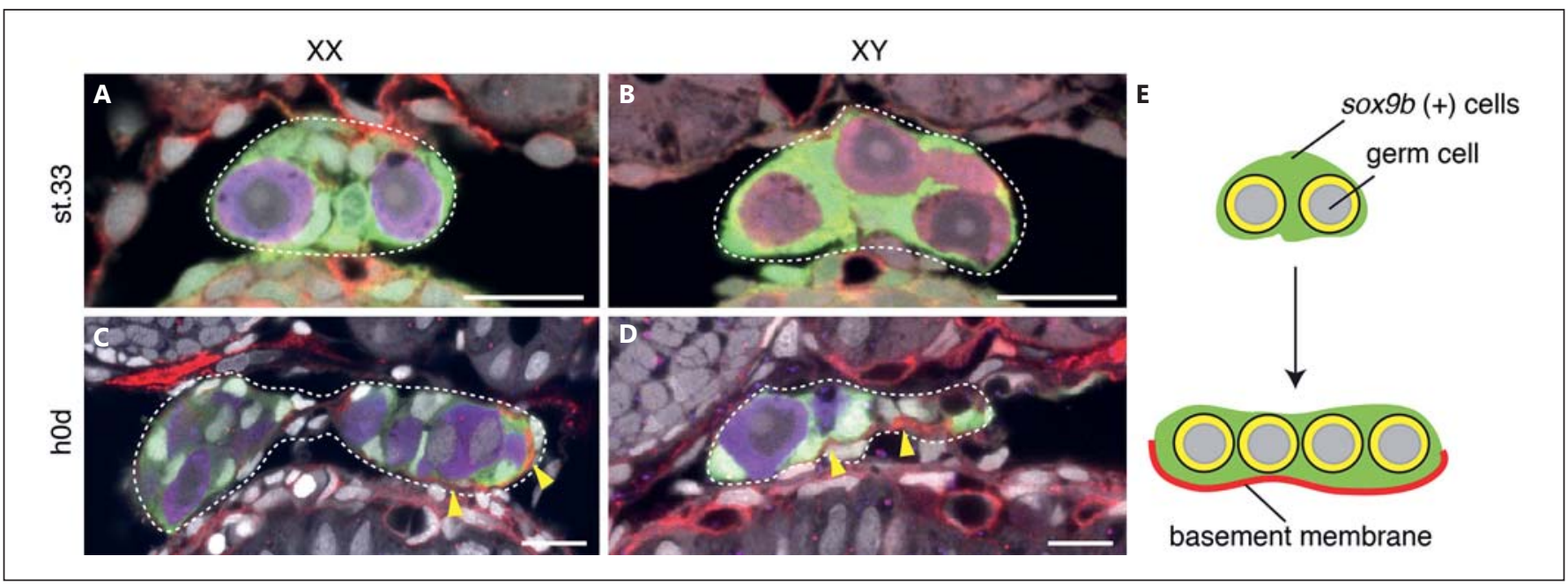

Fig. 2. Cross sections of the gonadal primordium in medaka. Dotted lines indicate location of the gonadal primordium. A, B The gonadal primordium mainly consists of sox $9 b$-expressing cells (green) surrounding germ cells (purple) in both XX and XY embryos at st.33. C, D A laminin-positive basement membrane (arrowheads) appears on a ventral side of the gonads at hatching stage (h0d). E Schematic representation of gonadal development from st. 33 to h0d. Scale bars $=10 \mu \mathrm{m}$.

these organs. During early sex differentiation, sox $9 b$-expressing cells surrounding germ cells are a main part of the gonadal primordium [Nakamura et al., 2008; Nishimura and Tanaka, 2014]. Therefore, we investigated when and how the basement membrane appears in the gonadal primordium to form the unit. The gonadal primordium in medaka is formed between the hindgut and coelomic epithelium at st.33 [Nakamura et al., 2006]. At this stage, sox $9 b$-expressing cells have already surrounded germ cells, but a laminin-positive basement membrane is absent in both $\mathrm{XX}$ and $\mathrm{XY}$ gonads (fig. 2A, B) [Nakamura et al., 2008]. At the early stage of gonadal sex differentiation (hatching stage), a laminin-positive basement membrane appears on the ventral side of the gonads in both XX and XY embryos (fig. 2C-E) [Saito et al., 2007]. sox $9 b$-null medaka exhibit disorganization of laminin deposits in gonadal somatic cells surrounding germ cells [Nakamura et al., 2012]. This may imply that the unit in the testes and ovaries arises via deposition of laminin in sox $9 b$-expressing cells close to the hatching stage.

Given that the unit originates from the gonadal primordium in both $\mathrm{XX}$ and $\mathrm{XY}$ embryos at the early stage of gonadal sex differentiation, the development and structure of ovaries and testes can be understood as follows: during ovarian development, the unit, together with epithelial cells, gives rise to the germinal epithelium. In addition, ovaries develop follicles in the stromal compart- ment. Therefore, ovaries can be viewed as an organ composed of a germinal epithelium with a well-developed stromal compartment. In foxl3 mutant ovaries, spermatogenesis occurs within the expanded germinal epithelium, forming a lobule-like structure. This observation allows us to speculate that the unit expands and gives rise to the lobule of the testes. In this regard, testis can be viewed as an expanded germinal epithelium where spermatogenesis occurs in an organized manner (fig. 1).

\section{Comparison of Gonadal Structures between Medaka and Amniotes}

In amniotes, including mammals, birds and reptiles, a gonadal primordium forms as a thickening of the coelomic epithelium on the surface of the mesonephros. The gonadal primordium can be regionalized into cortex and medulla (fig. 3) [Smith and Sinclair, 2004; DeFalco and Capel, 2009; Jiménez, 2009]. While the cortex develops by proliferation of the coelomic epithelium, the medulla is made up of cord structures (medullary cords) interspersed with mesenchymal cells (fig. 3). During ovarian development, oocyte growth occurs in the expanded cortex and medullary cords are fragmented. During testicular development, medullary cords expand and give rise to seminiferous tubules of the testes, and the cortex is re- 
Fig. 3. Fragmentation (ovary) and expansion (testis) of medullary cords in amniotes. The gonadal primordium in amniotes consists of 2 parts: cortex and medulla. During ovarian development, germ cells do not develop as stem cells but enter oogenesis. As a result, the cortex expands and becomes the place of follicle formation and growth, and medullary cords populated by sox9-expressing cells are fragmented. During testicular development, medullary cords expand with sox9-expressing cells to form seminiferous tubules that harbor germline stem cells, and the cortex reduces to become the tunica albuginea. Note that medullary cords are underlain by a basement membrane.

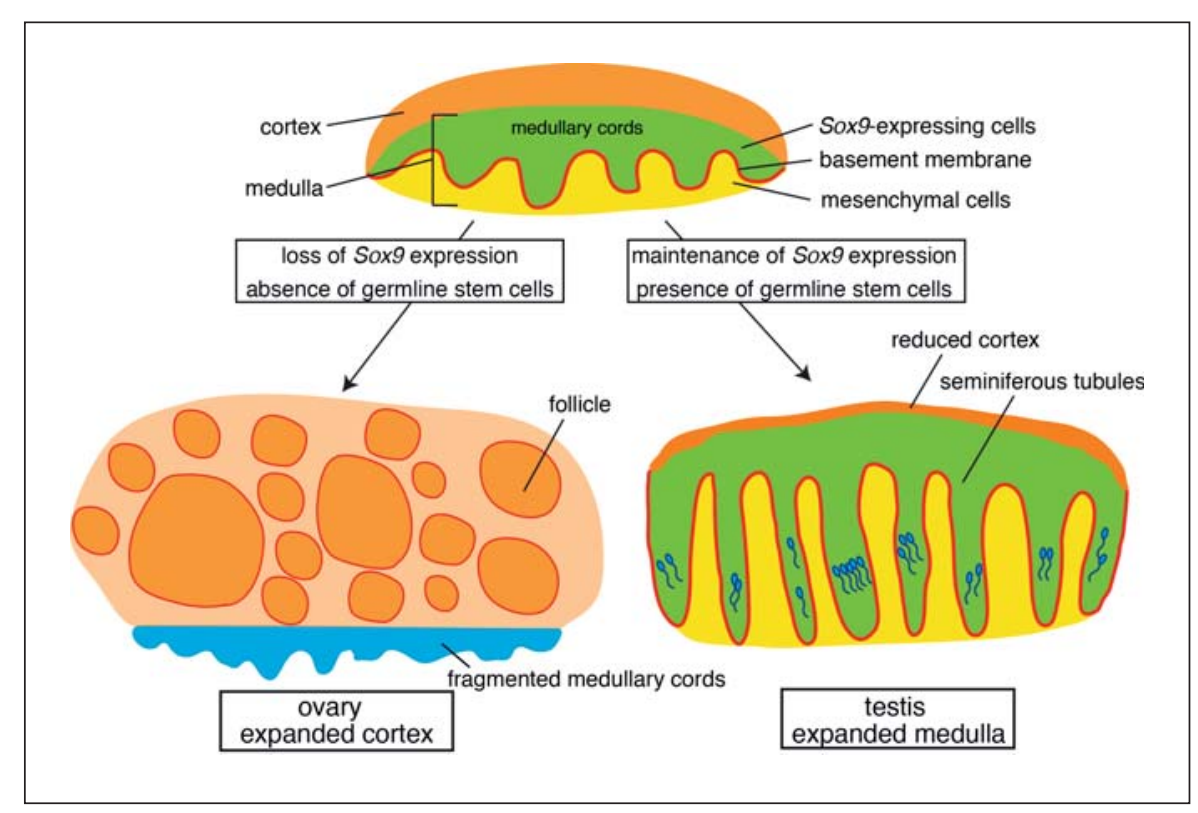

duced and becomes the tunica albuginea of the testes (fig. 3).

In mice, the medullary cords are not conspicuous in the gonadal primordium and appear only in male gonads after sex determination. However, this does not seem to represent the typical structure of the developing primordium. In other mammals, such as human and moles, as well as in birds and reptiles, medullary cords are present in the gonadal primordium of both males and females [Smith and Sinclair, 2004; DeFalco and Capel, 2009; Jiménez, 2009]. The medullary cords originate from ingression or invagination of coelomic epithelial cells and are underlain by the basement membrane (fig. 3) [Karl and Capel, 1998; Yao et al., 2004].

In amniotes, Sox9 is expressed in the medullary cords, but the signal disappears during ovarian development (fig. 3) [Barske and Capel, 2010; Kobayashi et al., 2005]. As Sox9 expression is downregulated, medullary cords degenerate in the ovaries [Barske and Capel, 2010]. Considering the fact that Sox 9 is essential for testis determination in mice [Vidal et al., 2001; Lavery et al., 2011], these results may suggest that for other amniotes, Sox9 is required for the maintenance of medullary cords and development of seminiferous tubules.

Conditional knockout of Sox 9 after testis differentiation indicates another function of Sox9. The depletion of Sox9 function at later stages results in the loss of most spermatogenic cells, including spermatogonial stem cells in mice [Barrionuevo et al., 2009]. In medaka, germline stem cells and sox $9 b$-expressing cells are present in both ovaries and testes [Nakamura et al., 2010]. Higher expression of sox $9 b$ is observed in the supporting cells enclosing germline stem cells/gonial cells than those surrounding germ cells at an advanced gametogenetic stage [Nakamura et al., 2011; Nishimura and Tanaka, 2014]. Furthermore, deletion of sox $9 \mathrm{~b}$ leads to the loss of germ cells in both males and females [Nakamura et al., 2012]. These results together suggest that the cells expressing Sox9 in amniotes and $s 0 x 9 b$ in medaka may have the common function of maintaining germline stem cells.

Regarding structural and functional similarity, the unit in the testes and ovaries in medaka, which is germ cells with sox $9 b$-expressing cells underlain by a basement membrane, may correspond to medullary cords in amniotes. On the basis of this assumption, gonadal structure in both amniotes and medaka could be explained uniformly. First, the unit in both amniotes and medaka expands and gives rise to seminiferous tubules or lobules of testes and provides a niche for germline stem cells. Second, in medaka ovaries, the unit is maintained as germinal cradles within the germinal epithelium to support germline stem cells not only for production of eggs but also for sperm. This idea is supported by observations in female foxl3 mutants.

In amniotes, especially in mammals, typical germline stem cells have not been found in ovaries [Nakamura et al., 2011; Spradling et al., 2011]. This would release the constraints of niche maintenance for germline stem cells 
in the ovary and is possibly related to the fragmentation of medullary cords during ovarian development. In this context, the medullary cords in amniotes may be an essential tissue that gives rise to niches for germline stem cells as well as seminiferous tubules.

\section{Conclusion}

Germ cells with sox $9 b$-expressing cells underlain by a basement membrane are structurally and functionally common units of testes and ovaries in medaka. On the basis of this unit, structural differences and similarities of testes and ovaries can be simply understood. It has been recently discovered that mature adult ovaries can be transdifferentiated into functional testes in several teleosts, including medaka [Paul-Prasanth et al., 2013], tilapia [Sun et al., 2014], and zebrafish [Takatsu et al., 2013] by inhibiting estrogen production. Testicular tissues have been suggested to originate from the germinal epithelium of ovaries [Paul-Prasanth et al., 2013], supporting the notion that the testis is an expanded version of the germinal epithelium within which spermatogenesis occurs in an organized manner. In this context, the common unit may also play an important role in the sex reversal process of natural hermaphroditic fish.

Furthermore, we would like to propose the possibility that the common unit in medaka is equivalent to the medullary cords of the gonadal primordium in amniotes. Characterization of other structural and functional units of testes and ovaries (e.g., the steroidogenic system) will provide further insights into another common structure of the gonadal primordium in both amniotes and teleosts.

\section{Acknowledgements}

This review was supported by a Grant-in-Aid for Scientific Research (A) (25251034) (M.T.) and a Grant-in-Aid for Research Activity Start-up (15H06838) (T.N.).

\section{Disclosure Statement}

The authors have no conflicts of interest to declare.

\section{References}

Aoki Y, Nakamura S, Ishikawa Y, Tanaka M: Expression and syntenic analyses of four nanos genes in medaka. Zoolog Sci 26:112-118 (2009).

Barrionuevo F, Georg I, Scherthan H, Lécureuil C, Guillou F, et al: Testis cord differentiation after the sex determination stage is independent of Sox 9 but fails in the combined absence of Sox9 and Sox8. Dev Biol 327:301-312 (2009).

-Barske LA, Capel B: Estrogen represses SOX9 during sex determination in the red-eared slider turtle Trachemys scripta. Dev Biol 341:305314 (2010).

Beer RL, Draper BW: nanos3 maintains germline stem cells and expression of the conserved germline stem cell gene nanos 2 in the zebrafish ovary. Dev Biol 374:308-318 (2013).

-DeFalco T, Capel B: Gonad morphogenesis in vertebrates: divergent means to a convergent end. Annu Rev Cell Dev Biol 25:457-482 (2009).

Grier HJ: Sperm development in the teleost Oryzias latipes. Cell Tissue Res 168:419-431 (1976).

Grier HJ: Cellular organization of the testis and spermatogenesis in fishes. Integr Comp Biol 21:345-357 (1981)
Grier HJ: The germinal epithelium: its dual role in establishing male reproductive classes and understanding the basis for indeterminate egg production in female fishes, in Proceedings of the Gulf and Caribbean Fisheries Institute, 53, pp 537-552 (2002).

Jiménez R: Ovarian organogenesis in mammals: mice cannot tell us everything. Sex Dev 3: 291-301 (2009).

Karl J, Capel B: Sertoli cells of the mouse testis originate from the coelomic epithelium. Dev Biol 203:323-333 (1998).

-Klüver N, Kondo M, Herpin A, Mitani H, Schartl M: Divergent expression patterns of Sox $9 \mathrm{du}-$ plicates in teleosts indicate a lineage specific subfunctionalization. Dev Genes Evol 215: 297-305 (2005).

Kobayashi A, Chang H, Chaboissier M-C, Schedl A, Behringer RR: Sox9 in testis determination. Ann NY Acad Sci 1061:9-17 (2005).

Kurokawa H, Saito D, Nakamura S, Katoh-Fukui Y, Ohta K, et al: Germ cells are essential for sexual dimorphism in the medaka gonad. Proc Natl Acad Sci USA 104:16958-16963 (2007).

Lavery R, Lardenois A, Ranc-Jianmotamedi F, Pauper E, Gregoire EP, et al: XY Sox 9 embryonic loss-of-function mouse mutants show complete sex reversal and produce partially fertile XY oocytes. Dev Biol 354:111-122 (2011).
Nakamoto M, Suzuki A, Matsuda M, Nagahama Y, Shibata N: Testicular type Sox9 is not involved in sex determination but might be in the development of testicular structures in the medaka, Oryzias latipes. Biochem Biophys Res Commun 333:729-736 (2005).

Nakamura S, Kobayashi D, Aoki Y, Yokoi H, Ebe $\mathrm{Y}$, et al: Identification and lineage tracing of two populations of somatic gonadal precursors in medaka embryos. Dev Biol 295:678688 (2006).

- Nakamura S, Aoki Y, Saito D, Kuroki Y, Fujiyama A, et al: Sox9b/sox9a2-EGFP transgenic medaka reveals the morphological reorganization of the gonads and a common precursor of both the female and male supporting cells. Mol Reprod Dev 75:472-476 (2008).

- Nakamura S, Kobayashi K, Nishimura T, Higashijima S, Tanaka M: Identification of germline stem cells in the ovary of the teleost medaka. Science 328:1561-1563 (2010).

Nakamura S, Kobayashi K, Nishimura T, Tanaka M: Ovarian germline stem cells in the teleost fish, medaka (Oryzias latipes). Int J Biol Sci 7: 403-409 (2011).

Nakamura S, Watakabe I, Nishimura T, Toyoda A, Taniguchi Y, Tanaka M: Analysis of medaka sox9 orthologue reveals a conserved role in germ cell maintenance. PLoS One 7:e29982 (2012). 
- Nishimura T, Tanaka M: Gonadal development in fish. Sex Dev 8:252-261 (2014).

Nishimura T, Sato T, Yamamoto Y, Watakabe I, Ohkawa $\mathrm{Y}$, et al: foxl3 is a germ cell-intrinsic factor involved in sperm-egg fate decision in medaka. Science 349:328-331 (2015).

-Parenti LR, Grier HJ: Evolution and phylogeny of gonad morphology in bony fishes. Integr Comp Biol 44:333-348 (2004).

- Paul-Prasanth B, Bhandari RK, Kobayashi T, Horiguchi R, Kobayashi Y, et al: Estrogen oversees the maintenance of the female genetic program in terminally differentiated gonochorists. Sci Rep 3:2862 (2013).
-Saiki A, Tamura M, Matsumoto M, Katowgi J, Watanabe A, Onitake K: Establishment of in vitro spermatogenesis from spermatocytes in the medaka, Oryzias latipes. Dev Growth Differ 39:337-344 (1997).

Saito D, Morinaga C, Aoki Y, Nakamura S, Mitani $\mathrm{H}$, et al: Proliferation of germ cells during gonadal sex differentiation in medaka: insights from germ cell-depleted mutant zenzai. Dev Biol 310:280-290 (2007).

Smith CA, Sinclair AH: Sex determination: insights from the chicken. BioEssays 26:120132 (2004).

-Spradling A, Fuller MT, Braun RE, Yoshida S: Germline stem cells. Cold Spring Harb Perspect Bil 3:a002642 (2011).
Sun LN, Jiang XL, Xie QP, Yuan J, Huang BF, et al: Transdifferentiation of differentiated ovary into functional testis by long-term treatment of aromatase inhibitor in Nile tilapia. Endocrinology 155:1476-1488 (2014).

Takatsu K, Miyaoku K, Roy SR, Murono Y, Sago $\mathrm{T}$, et al: Induction of female-to-male sex change in adult zebrafish by aromatase inhibitor treatment. Sci Rep 3:3400 (2013).

-Vidal VP, Chaboissier MC, de Rooij DG, Schedl A: Sox9 induces testis development in XX transgenic mice. Nat Genet 28:216-217 (2001).

-Yao HH, Dinapoli L, Capel B: Cellular mechanisms of sex determination in the red-eared slider turtle, Trachemys scripta. Mech Dev 121:1393-1401 (2004). 